\title{
Clinical Spectrum of Desquamative Inflammatory Vaginitis
}

\author{
Colleen Kennedy Stockdale
}

Published online: 2 September 2010

(C) Springer Science+Business Media, LLC 2010

\begin{abstract}
Desquamative inflammatory vaginitis (DIV) is a rare chronic clinical syndrome of unknown etiology characterized by profuse purulent vaginal discharge, diffuse exudative vaginitis, epithelial cell exfoliation, and pain. A diagnosis of DIV is often missed by even experienced practitioners owing to its rarity and its clinical and laboratory presentation similar to other inflammatory vulvovaginal disorders. Although DIV is difficult to treat and often requires long-term therapy for maintenance, successful therapy has been reported with topical steroids and clindamycin.
\end{abstract}

Keywords Desquamative · Inflammatory · Vaginitis ·

Vaginal discharge $\cdot$ Vaginal disease

\section{Introduction}

Chronic vaginitis symptoms are one of the most frequent reasons for patient visits to obstetrician-gynecologists. Despite the high prevalence of vaginitis symptoms, an estimated $30 \%$ of women may go without a diagnosis after a standard evaluation [1]. The inability to adequately control vaginitis symptoms as well as lack of a clearly defined diagnosis is frustrating for women, partners, and practitioners. Diagnosis requires careful history and physical evaluation (including office-based microscopy, culture, and histology) because clinical features associated

C. K. Stockdale $(\bowtie)$

Department of Obstetrics and Gynecology, University of Iowa, 200 Hawkins Drive,

Iowa City, IA 52242, USA

e-mail: colleen-stockdale@uiowa.edu with noninfectious and infectious causes of vaginitis are generally indistinguishable.

Desquamative inflammatory vaginitis (DIV) - also termed exudative vaginitis, hydrorrhea vaginalis, erosive vaginitis, or hemorrhagic vaginitis - is a rare but disabling condition that causes a profuse purulent vaginal discharge and severe vulvovaginal inflammation [1]. Features of DIV may be the result of noninfectious blistering disorders such as pemphigus vulgaris, lichen planus, and mucous membrane pemphigoid. Whether or not a distinct, separate entity of idiopathic DIV exists, or whether findings represent the spectrum of erosive lichen planus, remains controversial. For the purposes of this article, an idiopathic classification of DIV separate from erosive lichen planus is considered.

\section{Background/History}

The initial description of DIV is credited to Franken in 1954 as a chronic type of exudative vaginitis occurring in a 12-year-old girl who responded well to a local estrogen preparation [2]. Subsequent cases were presented by Scheffey et al. [2] (1956), Gray and Barnes [3] (1965), and Gardner [4] (1968), describing women with normal estrogen levels and features of exudative vaginitis and severe atrophic vaginitis: fragility, erythema, and copious discharge. However, many of the clinical features described by Gray and Barnes and by Gardner were noted to resemble vaginal lichen planus in later case reports and reviews by others [5-8]. More recently, evaluation of women with DIV not meeting the criteria for dermatologic conditions including erosive lichen planus has reintroduced the possibility of a separate, distinct diagnosis of DIV (idiopathic DIV). 


\section{Etiology}

The etiology of DIV remains unknown. DIV has been associated with erosive lichen planus, pemphigus vulgaris, and mucous membrane pemphigoid [1]. Recently, vitamin D deficiency has been proposed as an etiology of DIV based on clinical observations and a case report of a woman with refractory DIV symptoms and vitamin D deficiency that responded to pharmacologic vitamin D replacement [9]. Other proposed etiologies include unidentified microbial pathogen, diminished estrogen, autoimmune disorder, cellmediated immune activation (hyperimmune disorder), and possible genetic link [10, 11]. More likely, DIV represents a heterogeneous group of disorders with common clinical and microscopic characteristics [12].

\section{Epidemiology}

The epidemiology of DIV appears to vary significantly depending on the clinic population and possibly undefined clinician factors. DIV has been reported to occur in women of any age $[7,13]$, exclusively in premenopausal women $[4,14,15 \cdot]$, and to occur more frequently in postmenopausal women [16]. Although some investigators note that DIV often occurs in the presence of apparently normal ovarian function [4, 7, 13], others report that it is more frequently diagnosed in estrogendeficient older nonmenstruating women $[1,16]$. DIV was identified in $8 \%$ of women presenting with chronic vulvovaginal symptoms to a vulvar specialty clinic [16]. However, the population prevalence remains unknown. Although women with DIV were more likely to describe a previous diagnosis of vaginal infection, demographics were otherwise similar to friend controls in a case-control study [17].

Recent findings regarding women diagnosed with DIV were presented at the International Society for the Study of Vulvovaginal Disease 20th World Congress in September 2009. Reichman and Sobel [18•] noted that women with DIV were predominately Caucasian (despite a $60 \%$ African American clinic population), more likely to have thyroid disease and allergic diatheses, and more likely to respond to anti-inflammatory therapy based on a retrospective chart review of 98 women diagnosed with DIV. Similar demographic findings have been noted in other vulvar specialty clinics, but the rarity of DIV precludes rigorous research toward the better understanding of the epidemiology and etiology as well as optimal treatment options. A review of the English literature identified a single case-control epidemiology study, and two cohort studies. The remaining studies are case reports and case series.

\section{Pathogenesis}

Studies have failed to reveal a causal mechanism or specific microorganism for DIV [8]. Although the marked changes of DIV are relatively rare, milder findings of inflammatory vaginitis are not uncommon [19]. Inflammatory vaginitis not meeting criteria for DIV has also been termed "aerobic vaginitis" $[19,20]$.

The frequency of DIV in premenopausal, postmenopausal, and postpartum women and in those receiving antiestrogen therapy for endometriosis suggests that estrogen deficiency may play a role in the infectious process [8]. Alternatively, almost all women in one retrospective review received multiple courses of antibacterial and antimycotic agents and local remedies [8]. Thus, it is feasible that DIV represents an immunologic process precipitated by either a hypersensitivity reaction or antibacterial effects.

\section{Clinical Diagnosis}

Although DIV is a "clinical diagnosis," it is not easy to make the diagnosis of DIV because the clinical and laboratory findings are not unique. Clinical, colposcopic, and cytologic findings mimic those seen in women with trichomoniasis, severe atrophic vaginitis, and blistering dermatoses [4, 14]. The classic triad of DIV includes dyspareunia, increased vaginal discharge, and a sterile inflammatory vaginitis [13].

The diagnosis of DIV is based on clinical findings and findings on wet mount examination [14]. Diagnostic criteria include purulent vaginal discharge, leukocyte to epithelial cell ratio greater than 1:1 (in at least four high-power fields), and vaginal $\mathrm{pH}$ greater than 4.5, after excluding bacterial vaginosis, Neisseria gonorrhoeae, Chlamydia trachomatis, and Trichomonas vaginalis infection [8].

\section{Signs and Symptoms}

The most commonly reported symptom is the presence of an intractable, purulent vaginal discharge that has often persisted for years and is sometimes associated with vulvar soreness, burning, and dyspareunia [7, 8, 16]. Vulvovaginal itching may similarly be noted $[8,19]$.

Examination of the vagina is significant for erythema with marked inflammation, often with petechiae or punctate erosions (similar to the "strawberry cervix" associated with T. vaginalis infection) $[8,15 \bullet, 16]$. The presence of vaginal synechiae and stenosis has been identified in women with DIV [4, 7, 15•], although it is more suggestive of erosive lichen planus and some vulvovaginal experts believe that the presence of vaginal synechiae excludes a diagnosis of 
DIV $[10,21]$. DIV should be considered when women present for evaluation of dyspareunia. Nyirjesy [1] found that about half of the women with DIV rated their pain with intercourse on a visual analog scale similar to that of women with vestibulodynia. Thus, without a speculum examination, DIV may be misdiagnosed as a vulvar pain syndrome [13].

\section{Laboratory Diagnosis}

The distinguishing microscopic features of DIV include a marked increase in polymorphonuclear leukocytes (5-10 per epithelial cell) and immature epithelial parabasal cells $(>10 \%$ of total epithelial cells), along with a heavy coccoid vaginal flora and absence of long bacilli (lactobacilli) [8, $15 \cdot, 16]$, but no clue cells [14]. The vaginal $\mathrm{pH}$ is increased (typically 5.5 or greater). Gram staining shows an absence of lactobacilli and increased levels of gram-positive cocci $[8,13]$. Although there may be an increase in the presence of group B streptococci, repeated cultures for pathogenic bacteria, yeast, chlamydia, and viruses are negative $[8,13]$. This is similar to the microscopic and culture findings reported by Donders et al. [20] for aerobic vaginitis. Unlike bacterial vaginosis, clue cells and malodorous "fishy" discharge are absent in DIV.

Some investigators recommend biopsy to exclude other causes of desquamative inflammatory vaginitis before considering a diagnosis of (idiopathic) DIV [13]. However, otherwise unclassifiable cases of desquamative inflammatory vaginitis are often assigned a diagnosis of lichen planus in the setting of a nondiagnostic biopsy (limiting the usefulness of biopsy) [15•]. To evaluate specific histologic features of idiopathic DIV, biopsies were reviewed in 11 premenopausal women with clinical diagnoses of DIV demonstrating two patterns of inflammation: a lichenoid inflammatory pattern (falling short of the histologic criteria for lichen planus) in seven women, and a nonspecific mixed inflammatory infiltrate in four women [15•]. Whether the findings represent variants of the same process is unclear.

\section{Differential Diagnosis of DIV}

The differential diagnosis of desquamative inflammatory vaginitis includes dermatologic conditions such as linear immunoglobulin A disease, pemphigus vulgaris, mucous membrane pemphigoid, erosive lichen planus, and severe atrophic vaginitis [7]. The only infectious cause that needs to be ruled out is trichomoniasis. This may be accomplished by microscopy, culture, or a point-of-care diagnostic test such as the OSOM Trichomonas Rapid Test (Genzyme Diagnostics, Cambridge, MA).
Patients with dermatologic conditions may have evidence of extragenital disease; therefore, a complete history and physical examination should be performed. It is essential to examine the mouth and eyes to differentiate DIV from any similarly presenting mucous membrane disorders (eg, mucous membrane pemphigoid, erosive lichen planus). Altered flora patterns of inflammatory vaginitis and desquamative inflammatory vaginitis may be observed in conjunction with erosive or blistering dermatologic conditions [19] and atrophic vaginitis [14]. In these cases, the clinical and laboratory abnormalities resolve with treatment of the underlying condition [19]. Alternately, inflammatory vaginitis may be the result of coexisting diagnoses and may require concurrent treatment. Improvement in symptoms with estrogen therapy helps to distinguish atrophic vaginitis from DIV in menopausal and estrogen-deficient women [7].

Women with DIV note discharge as the prominent symptom, whereas those with lichen planus report soreness, pain, and dyspareunia rather than discharge [7].Unlike erosive lichen planus, adhesion formation is generally not seen with DIV [10, 21]. As previously noted, DIV may be misdiagnosed as a vulvar pain syndrome without a vaginal speculum examination [13]. Biopsy to confirm a dermatologic or mucous membrane etiology of DIV is valuable when classic criteria are present for histopathologic examination and immunofluorescence studies.

Pemphigus vulgaris is a chronic, acantholytic, blistering disease that usually manifests initially at mucosal sites. It has a characteristic histologic appearance of supranasal acantholysis, direct immunofluorescence shows deposition of intercellular immunoglobulin $\mathrm{G}$, and there are circulating autoantibodies [22].

Mucous membrane pemphigoid is a rare, chronic, blistering disease of the mucous membranes and skin that results in permanent scarring of the affected area. Histology shows subepithelial bulla formation with a sparse lymphohistiocytic infiltrate. Deposition of linear IgG, IgA, and $\operatorname{IgM}$ with complement 3 in the basement membrane zone is found with direct immunofluorescence [22].

Linear immunoglobulin A disease is a cutaneous, vesiculobullous disorder that occurs in young people and has a slight female predominance. It has a linear deposition of immunoglobulin A in the basement membrane zone [22].

\section{Risk Factors}

In a case-control study, women with DIV were older, more likely married, and significantly more likely to report vaginal symptoms, including discharge, pain, and dyspareunia, as well as history of vulvovaginal candidiasis, bacterial vaginosis, pelvic inflammatory disease, use of oral contraceptives, use of hormone replacement therapy, 
and laparoscopic procedures than either friend or population controls [17]. Whether the presence of vaginal symptoms including a history of yeast and bacterial vaginitis are a marker or a risk factor for DIV is not clear.

\section{Treatment of DIV}

Treatment of DIV is primarily anecdotal and largely inadequate. Because it is an inflammatory vaginitis by definition, the approach to treatment has been the reduction of vaginal inflammation, allowing restoration of normal sexual function [13]. Regimens using intravaginal steroid, either alone or in combination with clindamycin, have emerged as the first-line treatment for DIV. However, some individuals require hormone replacement therapy for sustained symptom relief, whereas others fail to respond to all interventions [9]. Unlike other vulvovaginal disorders, clinical symptoms typically improve before the vaginal wall epithelium normalizes fully [15•]. Reported treatment regimens for DIV are presented in Table 1.

Of 51 women with DIV treated using intravaginal clindamycin $2 \%$, the overall improvement was noted to be $90 \%$, with symptom relapse occurring in $29 \%$ [8]. In a smaller study using oral clindamycin, 9 of $11(82 \%)$ improved following treatment with oral clindamycin, $150 \mathrm{mg}$ twice weekly, intravaginal clobetasone propionate on alternate days, and fluconazole, $150 \mathrm{mg}$ once weekly (to prevent yeast infections) for 4 to 6 weeks [15•]. Because topical clindamycin functions as both an anti-inflammatory agent and antibacterial agent, its role in the treatment of DIV remains controversial [15•].

Although postmenopausal patients with DIV did not demonstrate improvement in their symptoms with local estrogen therapy alone [15•], the addition of exogenous estrogen after initial treatment with intravaginal clindamycin has been suggested in an effort to decrease the likelihood of symptom recurrence [16]. It is believed that the addition of vaginal estrogen creates an environment more favorable to the growth of lactobacilli [11].

\section{Recurrent DIV}

In most women with DIV, treatment response is inconsistent, recurrence is common, and maintenance therapy is required to reduce symptoms. Treatment options for recurrent DIV are also based on anecdotal recommendations and include repeating a previous successful regimen after verification of diagnosis (ie, rule out other cause of DIV) and reducing the frequency of treatment to the lowest dose that maintains relief of symptoms. Consideration of maintenance therapy using intravaginal steroid (eg, hydrocortisone, 25-mg suppositories) twice weekly is recommended to control waxing and waning symptoms [21]. Alternatively, local estrogen replacement or intermittent use of clindamycin may be used separately or in combination for long-term symptom control.

\section{Conclusions}

DIV is a poorly understood and undiagnosed chronic clinical syndrome of unknown etiology characterized by profuse vaginal discharge, diffuse exudative vaginitis, epithelial cell exfoliation, and pain. Despite the limited understanding of the etiology and treatment of DIV, it is well established that women with chronic vaginitis symptoms, including DIV, report a significant negative impact on their quality of life. DIV has been identified in $8 \%$ of women presenting to a vulvar vaginal disease clinic for evaluation of chronic vaginitis

Table 1 Treatments reported for desquamative inflammatory vaginitis

\begin{tabular}{|c|c|c|}
\hline \multirow[t]{2}{*}{ Topical steroids } & Hydrocortisone, 25-mg intravaginal suppository ${ }^{\mathrm{a}}$ & Nightly for 2-4 wk; reduce frequency for maintenance \\
\hline & $10 \%$ hydrocortisone foam intravaginal & $\begin{array}{l}2-3 \text { times daily for } 2 \mathrm{wk} \text {; consider prolonged } \\
\text { course or maintenance }[21]\end{array}$ \\
\hline \multirow[t]{2}{*}{ Topical antibiotics } & Clindamycin, 200-mg suppositories intravaginal & Nightly for $2-4 \mathrm{wk}[8,10]$ \\
\hline & $2 \%$ clindamycin cream intravaginal & $5 \mathrm{~g}$ nightly for $2-8 \mathrm{wk}[10,11,18 \bullet]$ \\
\hline \multirow[t]{4}{*}{ Combined therapy } & $\begin{array}{l}2 \% \text { clindamycin cream alternating with hydrocortisone, } \\
25-\mathrm{mg} \text { intravaginal suppository }{ }^{\mathrm{a}}\end{array}$ & Alternate nightly for 14 nights \\
\hline & $\begin{array}{l}\text { Hydrocortisone, } 100 \mathrm{mg} / \mathrm{g} \text { in clindamycin } 2 \% \text { cream } \\
\text { base intravaginal }^{\mathrm{a}}\end{array}$ & Insert $5 \mathrm{~g}$ every other night for 14 doses ( 28 nights). \\
\hline & $\begin{array}{l}2 \% \text { clindamycin cream ( } 1 \text { applicator) combined with } \\
\text { hydrocortisone, } 25-\mathrm{mg} \text { intravaginal suppository }{ }^{\mathrm{a}}\end{array}$ & Insert both every other night for 14 doses ( 28 nights) \\
\hline & $\begin{array}{l}\text { Hydrocortisone, } 100 \mathrm{mg} / \mathrm{g} \text { in clindamycin } 2 \% \text { cream base } \\
\text { (compounding pharmacy only) }\end{array}$ & $\begin{array}{l}\text { Insert } 5 \text {-g applicator every other night for } 14 \text { doses } \\
\text { (28 nights) }\end{array}$ \\
\hline Estrogen & Consider addition of local (intravaginal estrogen) & $\begin{array}{l}\text { Use recommended manufacturer dose } 1-2 \text { nights } \\
\text { weekly }[11,16]\end{array}$ \\
\hline
\end{tabular}

${ }^{\mathrm{a}}$ Recommendation not published in literature; based on anecdotal evidence/lecture 
symptoms, and should be considered in the evaluation of women presenting for chronic vaginitis symptoms, including dyspareunia. DIV likely represents a heterogeneous disorder with a similar clinical presentation. The diagnosis of DIV is based on the presence of purulent vaginal discharge, increased leukocyte to epithelial cell ratio greater than 1:1 and vaginal $\mathrm{pH}$ greater than 4.5, after excluding infectious etiologies. Although difficult to treat, successful therapy has been reported with topical steroids and clindamycin [13].

Disclosure No potential conflict of interest relevant to this article was reported.

\section{References}

Papers of particular interest, published recently, have been highlighted as:

- Of importance

1. Nyirjesy P, Peyton C, Weitz MV, et al.: Causes of chronic vaginitis. Obstet Gynecol 2006, 108:1185-1191.

2. Scheffey LC, Rakoff AE, Lang WR: An unusual case of exudative vaginitis (hydrorrhea vaginalis) treated with local hydrocortisone. Am J Obstet Gynecol 1956, 72:208-211.

3. Gray LA, Barnes ML: Vaginitis in women, diagnosis and treatment. Am J Obstet Gynecol 1965, 92:125-127.

4. Gardner HL: Desquamative inflammatory vaginitis: a newly defined entity. Am J Obstet Gynecol 1968, 102:1102-1105.

5. Lynch P: Desquamative inflammatory vaginitis with oral lichen planus. Presented at the Second International Congress of the International Society for the Study of Vulvar Disease. Key Biscayne, Florida: January 9-11, 1975.

6. Edwards L, Friedrich EG: Desquamative vaginitis: lichen planus in disguise. Obstet Gynecol 1988, 71:832-836.

7. Oates JD, Rowen D: Desquamative inflammatory vaginitis. A review. Genitourin Med 1990, 66:275-279.
8. Sobel JD: Desquamative inflammatory vaginitis: a new subgroup of purulent vaginitis responsive to topical $2 \%$ clindamycin therapy. Am J Obstet Gynecol 1994, 171:1215-1220.

9. Peacocke M, Djurkinak E, Thys-Jacobs S: Treatment of desquamative inflammatory vaginitis with vitamin D: A case report. Cutis 2008, 81:75-78.

10. Edwards L: Desquamative inflammatory vaginitis. In Genital Dermatology Atlas. Edited by Edwards L. Philadelphia: Lippincott Williams \& Wilkins; 2004:237-240.

11. Ledger WJ, Witkin SS: Desquamative inflammatory vaginitis. In Vulvovaginal Infections. Edited by Ledger WJ, Witkin SS. London: Manson Publishing; 2007:49-52.

12. Lev-Sagie A, Nyirjesy P: Treatment of desquamative inflammatory vaginitis (DIV) with intravaginal $2 \%$ clindamycin cream: a long term follow-up. J Low Genit Tract Dis 2009, 13(5 Suppl):S2.

13. Murphy R: Desquamative inflammatory vaginitis. Dermatol Ther 2004, 17:47-49.

14. Paavonen J: Desquamative inflammatory vaginitis. Infect Dis Obstet Gynecol 1996, 4:257.

15. - Murphy R, Edwards L: Desquamative inflammatory vaginitis, what is it? J Reprod Med 2008, 53:124-128. The authors report the histopathology findings of idiopathic DIV.

16. Nyirjesy P: Postmenopausal vaginitis. Curr Infect Dis Rep 2007, 9:480-484.

17. Newbern EC, Foxman B, Leaman D, Sobel JD: Desquamative inflammatory vaginitis: an exploratory case-control study. Ann Epidemiol 2002, 12:346-352.

18. - Reichman R, Sobel JD: Desquamative inflammatory vaginitis (DIV): twelve year experience with one hundred and thirty patients - new insights. J Low Genit Tract Dis 2009, 13(5 Suppl):S2. The authors presented preliminary findings on the largest cohort of women with DIV.

19. Fowler RS: Expansion of altered vaginal flora states in vaginitis to include a spectrum of microflora. J Reprod Med 2007, 52:93-99.

20. Donders GGG, Vereecken A, Bosmans E, et al.: Definition of a type of abnormal vaginal flora that is distince from bacterial vaginosis: aerobic vaginitis. BJOG 2002, 109:34-43.

21. Dennerstein G, Scurry J, Brenan J, et al: Desquamative inflammatory vaginitis. In The Vulva and Vagina Manual. Edited by Dennerstein G, Scurry J, Brenan J, et al. Australia: Gynederm Publishing; 2005:170-172.

22. Jacobson M, Krumholz B, Franks A: Desquamative inflammatory vaginitis. J Reprod Med 1989, 34(9):647-650. 